\title{
Calcium stores, SERCAs and PMCA in human platelets stimulated by thrombin: A reinterpretation
}

\author{
Alfonsas Juška \\ Vilniaus Gedimino technikos universitetas, Saulètekio al. 11, 10223 Vilnius, Lithuania. \\ Tel: 370 5-2370561, E-mail: Alfonsas@vgtu.lt
}

doi:10.5618/bio.2011.v1.n1.5 || Received: 03-11-2011, Accepted: 15-11-2011, Available online: 14-11-2011

\begin{abstract}
It has been found on the basis of experimental data by Redondo and co-workers (Cell. Signal. 2008) that under the conditions of the experiments thrombinstimulated discharge of DTS is negligible compared to that of the acidic stores. There is good reason to believe that SERCA is activated by unbinding calcium ion(s) from the binding site(s) located on the inner (stores-facing) side of the (macro)molecule. Serious errors in the quoted paper are pointed out: 1) The fundamental difference between calcium quantity or concentration (essentially non-temporal value) and its fluxes (essentially temporal values) has been ignored throughout. 2) Calcium extrusion by PMCA has not been taken into account when analyzing thrombin-stimulated increase in cytosolic calcium concentration. 3) Use of integration to evaluate calcium entry or its amount in the stores is erroneous. 4) A single graph in each figure of the quoted paper (Figs. 1F - 4F) presented to characterize $\mathrm{Ca}^{2+}$ remaining in the stores (nM.s) cannot reflect calcium content in the stores of both types, their properties being different.
\end{abstract}

Keywords: Thrombin; Calcium stores; Calcium extrusion; Sarco-endoplasmic reticulum calcium ATPase; Plasma membrane calcium ATPase; Cytosolic calcium concentration; Dynamics of calcium concentration.

Abbreviations: PMCA, plasma membrane calcium ATPase; SERCA, sarco-endoplasmic reticulum calcium ATPase; DTS, dense tubular system.

\section{Introduction}

Control of cytosolic free calcium concentration is vital for a large number of cellular processes. Calcium concentration in cytosol is $\sim 50-100 \mathrm{nM}$ while in various cellular compartments the concentration is $\sim 2$ orders higher than that in cytosol. It is clear that cytosolic calcium concentration is maintained or changed depending on calcium fluxes into (passive) or out of (active) cytosol (see, e.g. [1,2]). Calcium flux out of the stores into cytosol is controlled by various physiological agonists. The action of the agonists causes changes in permeability of endomembranes for calcium ions. While the immediate result of action of the agonists is (bio)chemical, calcium fluxes are physical processes. The system of the fluxes and concentrations together with the mechanisms involved in control of those fluxes, therefore, has to be considered as a physical system. Concerning the biochemical pathways and mechanisms of calcium influx into and its elimination from platelet cytosol, a large volume of experiments has been performed up to date (see e.g. [3-5]). As a by-product of biochemical and physiological studies, these investigations provide valuable data related to the dynamics of the fluxes.

Information concerning the activities of PMCA [4]), the SERCAs [6] in platelets whose SERCAs are inhibited during the experiments, is rather detailed. It is less definite when platelets are stimulated by the agonists. Experimental investigation by Redondo and co-workers [7] provides information concerning the effect of thrombin; the time course of calcium content in the stores presented in the paper [7], however, cannot be considered accurate. In view of overwhelming complexity of the pathways of calcium fluxes in cells (see, e.g. [1]) and the variety of mechanisms involved [2], it is clear that any insight into the dynamics of calcium fluxes in platelets (and the mechanisms responsible for the fluxes) is not possible without application of adequate methods of data analysis (including mathematical modeling); reasonable simplification of real biological systems is necessary, however, to make modeling feasible and practicable.

The aim of this work, therefore, was to analyze the experimental data concerning cytosolic calcium concentration in platelets suspended in calcium-free medium and treated with a low concentration of a physiological agonist thrombin [7] in view of better understanding of the mechanisms involved in the maintenance and control 
of cytosolic calcium concentration. The known models concerning calcium fluxes were modified here to take into account various factors related to the mechanisms in question.

\section{Methods}

Standard software was used. The curves taken from PDF files were digitized with UN-SCAN-IT. Simple Visual Basic macro functions for Microsoft Excel [8] were used to numerically solve differential equations.

\section{Results and Discussion}

Experimental data of time-course of calcium concentration in cytosol of platelets suspended in calcium-free medium and stimulated by low concentration of thrombin were taken from the paper by Redondo and co-workers [7]. The data corresponding to the 4 possible combinations of states of SERCA2 $b$ and SERCA3 (active or inhibited) together with the models are presented in Fig. 1. The modeling is based on widely accepted assumptions concerning the mechanisms leading to the above time-course; it is aimed to elucidate, if possible, the details of the above mechanisms. It should be noted that the time-course of the concentration does not reflect those of calcium fluxes directly, but via integration of the latter $[9,10]$, making any inference concerning the fluxes less accurate than the primary data.

It is clear that cytosolic calcium concentration of platelets contained in calcium-free medium at any moment is the result of several calcium fluxes: its influx from calcium stores because of leakage via the endomembrane and (if stimulated) due to passage of the ions via the channels in the membrane and its extrusion by PMCA as well as calcium re-uptake back into the stores by SERCAs (see Fig. 2). It should be noted that the arithmetical operations of addition and subtraction are applicable to the fluxes rather than to concentrations as sometimes is assumed $[4,11]$. For simplicity, the rates of rise and decline in PMCA activity are supposed to be identical $[9,10]$, the corresponding equation being presented in the quoted papers.

It can be seen from Fig. 1 that the rise in the concentration resulting from stimulation by thrombin follows a quite clear lag. The lag, presumably, is related to that of rise in the permeability for calcium ions of the endomembranes. The additional (to the leakage) permeability caused by thrombin can be assumed to be pulse-like:

$$
p=\gamma^{4} t^{3} \exp (-4 \gamma t)
$$

where $\gamma$ is as defined in Table 1. It is clear that Eq. (1) is applicable (with corresponding parameters) to the endomembranes of the stores of both types. The rise and decline of the pulse depend on the properties of both thrombin and the pathway of stimulation of the change (thrombin degradation and stimulation and desensitization of the corresponding pathway). The parameter of the pulse is its mean duration $(1 / \gamma)$, the power of $\gamma(=4)$ is arbitrary, that of $t$ is lower by 1 , the multiplier in the exponential term $(=4)$ is the same as the power of $\gamma$ (manuscript in preparation).

To model the time-course of cytosolic calcium concentration resulting from store discharge, the SERCAs being inhibited, System (6) presented in [12] can be used. Neither calcium contents in the stores nor parameters characterizing the leakage from the stores of both types being known nor being possible to estimate from the data presented by Redondo and coworkers [7], the (relative) volumes of the stores unknown, it remained to take the lacking parameters arbitrarily (the volumes), or from other paper [6]; that may lead to inaccuracies: the conditions of the experiments as well as platelet preparations nominally being the same, may differ considerably, leading to the differences in the parameters. The results of modeling are presented in Fig. 1A which seem quite acceptable.

When SERCAs are not inhibited, it is necessary to take into account their activity. It is clear that it depends on the difference between the initial and current calcium concentrations in the stores, i.e.

$$
E_{\text {SERCA }}=\alpha\left(C_{0}-C\right)
$$

where $\alpha$ and $C_{0}$ are as defined in Table 1. Eq. (2) is valid (with corresponding parameters) for the both SERCAs. Lowering calcium concentration in the stores (reducing term $C$ in the above equation) corresponds to unbinding calcium ion(s) from the binding site(s) of the SERCA (macro)molecule facing the stores; that causes increase in the activity of SERCA. Expression (2), presumably, can be non-linear, parameter $\alpha$ depending on calcium concentration in the stores. Taking into account possible non-linearity, however, is bound to introducing more arbitrary parameters, and that does not seem to make sense.

Using Eqs. (1) and (2) and modifying System (6) from [12] for one of SERCAs inhibited, the other being active, leads to 


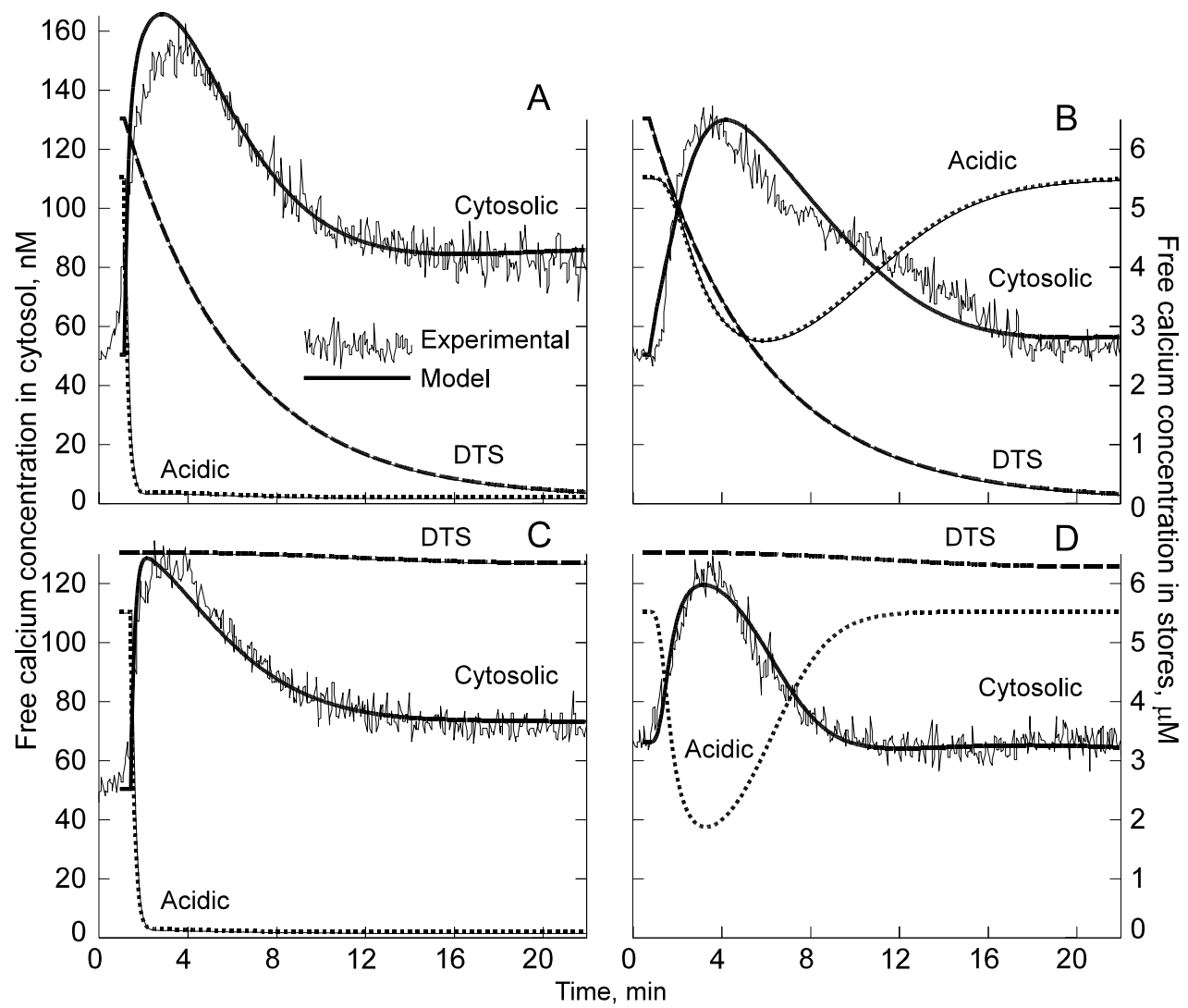

Fig.1. Dynamics of calcium concentration in cytosol of platelets and those in calcium stores resulting from stimulation by thrombin $(0.01 \mathrm{U} / \mathrm{mL})$. A, both SERCAs inhibited. B, SERCA2b inhibited, SERCA3 active. C, SERCA2b active, SERCA3 inhibited. D, both SERCAs active. The data of four to six experiments are taken from [7].

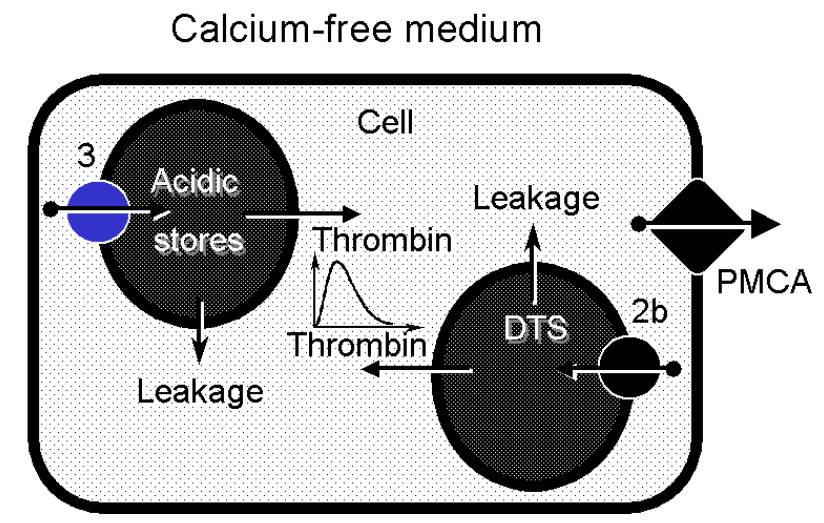

Fig.2. Schematic representation of a platelet contained in calcium-free medium, calcium stores, pathways and mechanisms of calcium fluxes. Only the pathways and mechanisms considered relevant are depicted here. Dots on the arrows' tails are intended to symbolize the "handling" of calcium ions one by one by the ATPases, the dotless arrows symbolizing the passive leakage or stimulated flow of the ions from the stores; 'Thrombin' means stimulated permeability of membranes of the stores, '2b', SERCA2b, '3', SERCA3. 


$$
\left\{\begin{array}{l}
\frac{d C}{d t}=-\left(\alpha+\varepsilon p_{\mathrm{DTS}}\right)(C-c) \\
\left.\frac{d Z}{d t}=\beta\left(Z_{0}-Z\right)-\zeta p_{\text {acidic }}\right)(Z-c) \\
\frac{d c}{d t}=\mathrm{\imath}\left(\alpha+\varepsilon p_{\mathrm{DTS}}\right)(C-c)-\kappa \beta\left(Z_{0}-Z\right) \\
+\kappa \zeta p_{\text {acidic }}(Z-c)-E_{\mathrm{PMCA}} \lambda^{2} t \exp (-\lambda t)
\end{array}\right.
$$

where $\varepsilon, \zeta, \imath, \kappa, \lambda$ and $E_{\mathrm{PMCA}}$ are as defined in Table 1.

Solutions of the above system (SERCA2b inhibited, SERCA3 active) together with experimental data are presented in Fig. 1B, its parameters being presented in Table 1. The results of the modeling seem quite acceptable.

Solutions of the remaining 2 variants of System (3) together with experimental data are presented in Figs. 1C and D, the parameters being presented in Table 1. Quite unexpectedly, the discharge of DTS in Figs. 1C and $\mathrm{D}$ is hardly noticeable, while that of the acidic stores is pronounced. It should be noted that thrombin concentration used $(0.01 \mathrm{U} / \mathrm{mL}$ [7]), presumably, can be considered to be physiological or close to it, while the model compatible with the data and based on reasonable assumptions suggests that under these conditions mainly the acidic stores rather than DTS are affected, the discharge of the former being pronounced while that of DTS is hardly noticeable (Figs. 1C and D). It follows, therefore, that under physiological conditions the acidic stores rather than DTS play the major role in thrombin-stimulated calcium influx into cytosol from the stores, the role of DTS being negligible. The difference arises, presumably, both from the difference in the permeabilities of the endomembranes of the stores and that of the activities of SERCA2b and SERCA3. The finding of the roles of DTS and acidic stores seems to be quite unexpected. (The discharge of DTS being negligible, the estimate of the duration of the pulse of increase in the endomembrane permeability, or parameter $\gamma$, cannot be considered accurate.)

By the end of experiments (Fig. 1D) calcium influx from the stores has to be low enough to keep cytosolic calcium concentration low. There is almost no calcium influx from the acidic stores by that time, the pulse of calcium efflux from these stores being short because of high activity of SERCA3. The activity of SERCA2b being rather low, the duration of calcium efflux from DTS is rather long. The duration of the efflux could be shortened by increasing calcium re-uptake back into the stores by SERCA2b (i.e. making expression (2) non-linear); that is inevitably bound up, as mentioned above, to introducing more arbitrary parameters. Of course it should be kept in mind that the dynamics of calcium concentration in the stores presented in Fig. 1 might slightly differ (because of arbitrary parameters of the models) resulting in almost identical (possible differences being non-detectable) dynamics of cytosolic calcium concentration. Nonetheless, the dynamics of calcium content in the stores of both types differ considerably from those presented in the quoted paper by Redondo and co-workers [7]. Serious errors in this paper make some graphs and the authors' conclusions incorrect, and experimental data have to be reinterpreted.

The following points concerning the article by Redondo and co-workers [7] have to be noted:

1. The fundamental difference between calcium quantity or concentration (observed experimentally, an essentially non-temporal value) and its fluxes (not observable directly, resulting from the activity of (molecular) mechanisms, essentially temporal values) has been ignored throughout.

2. Calcium extrusion by PMCA has not been taken into account when analyzing thrombin-stimulated increase in cytosolic calcium concentration.

3. The immediate use by the authors of integration to evaluate calcium entry or its amount in the stores is erroneous, because the integration over time results in a quantity whose dimension is [Concentration] $\times$ [Time] (e. g., $\mathrm{nM} \times \mathrm{s}$ ) and cannot be interpreted as just concentration (appropriate to characterize the content); division of the integral by the interval of integration [13] results in the temporal average.

4. A single graph in each figure (Figs. $1 \mathrm{~F}-4 \mathrm{~F}$ ) presented to characterize $\mathrm{Ca}^{2+}$ remaining in the stores (nM.s) [7] cannot characterize calcium content in the stores of both types, their properties being different (see [6]).

Analysis of experimental data does not suggest immediate involvement of SERCAs in regulation of SOCE.

Investigation of the dynamics of calcium concentration in the stores of any type separately (the stores of the other type being pre-discharged, see [6]) in combination with the experiments enabling to take into account calcium extrusion by PMCA [14] would provide more valuable information. It could be seen, e.g., whether thrombin-stimulated discharge of DTS is less pronounced than that of the acidic stores.

The points raised above concern the interpretation of experimental data which provide valuable information on calcium fluxes in the cells. Although variation rather 
than similarity of the properties of the cells (and corresponding parameters) in different experiments are expected, those properties, as follows from the analysis, are rather similar. Indeed, as seen from Table 1, most estimates of a parameter (except for efficacy of PMC) are almost the same for all 4 experiments.

Table 1. Parameters of model curves (Fig. 1) estimated ${ }^{\mathrm{a}-\mathrm{e}}$ as a result of fitting to experimental data [7]

\begin{tabular}{|c|c|c|c|c|c|}
\hline \multirow[t]{2}{*}{ Parameter } & \multirow{2}{*}{$\begin{array}{l}\text { Nota- } \\
\text { tion }\end{array}$} & \multicolumn{4}{|c|}{ Estimate } \\
\hline & & A & $\mathrm{B}$ & $\mathrm{C}$ & $\mathrm{D}$ \\
\hline Initial cytosolic calcium concentration, $\mathrm{nM}$ & $c_{0}$ & 50 & 50 & 50 & 66 \\
\hline Relative rate of discharge of DTS stimulated by thrombin, $\min ^{-1}$ & $\varepsilon$ & 8 & 8 & 6 & 8 \\
\hline $\begin{array}{l}\text { Reciprocal of mean duration of the pulse of DTS membrane } \\
\text { permeability, } \mathrm{min}^{-1}\end{array}$ & $\gamma$ & 0.05 & 0.05 & 0.05 & 0.05 \\
\hline $\begin{array}{l}\text { Relative rate of discharge of acidic stores stimulated by } \\
\text { thrombin, } \min ^{-1}\end{array}$ & $\zeta$ & 2000 & 2000 & 2000 & 2000 \\
\hline $\begin{array}{l}\text { Reciprocal of mean duration of the pulse of membrane } \\
\text { permeability of the acidic stores, } \mathrm{min}^{-1}\end{array}$ & $\delta$ & 0.3 & 0.15 & 0.3 & 0.3 \\
\hline Relative rate of PMCA transitions, $\mathrm{min}^{-1}$ & $\lambda$ & 0.36 & 0.35 & 0.36 & 0.4 \\
\hline Efficacy of PMCA, $10^{6}$ ions per cell & $E_{\mathrm{PMCA}}$ & 300 & 250 & 66 & 6 \\
\hline
\end{tabular}

${ }^{\text {a }}$ Relative volumes of DTS $\left(\mathfrak{l}=V_{\mathrm{DTS}} / V_{\text {cytosol }}\right)$ and acidic stores $\left(\kappa=V_{\text {acidic }} / V_{\text {cytosol }}\right)$ were assumed to be 0.04 and 0.015

${ }^{\mathrm{b}}$ Initial calcium concentration in DTS $\left(C_{0}\right)$ was assumed to be $6.5 \mu \mathrm{M}$

${ }^{\mathrm{c}}$ Initial calcium concentration in acidic stores was assumed to be $5.5 \mu \mathrm{M}$

${ }^{\mathrm{d}}$ Relative rate of calcium ions leakage from DTS was taken from $[6]\left(\alpha=0.2 \mathrm{~min}^{-1}\right)$

${ }^{\mathrm{e}}$ Relative rate of the leakage from the acidic stores was taken from $[6]\left(\beta=6 \mathrm{~min}^{-1}\right)$

\section{Conclusions}

Analysis of the pathways and mechanisms controlling calcium fluxes and modeling the thrombin-stimulated discharge of calcium stores in human platelets can be summarized as follows:

1. The fundamental difference between calcium quantity (essentially non-temporal value) and its fluxes (essentially temporal values) cannot be ignored.

2. Calcium extrusion by PMCA has to be taken into account when investigating any change in cytosolic calcium concentration.

3. It should be kept in mind that integration of any function over time leads to change of the dimension making it different from that of the original function.

4. Under conditions of the experiments [7] thrombinstimulated discharge of DTS is negligible in comparison with that of the acidic stores.

5. There is good reason to believe that SERCA is activated by unbinding calcium ion(s) from the binding site(s) located on the inner (stores-facing) side of the (macro)molecule.

\section{Appendix A. Supplementary data}

Supplementary data to this article can be found online at http://ccaasmag.org/bio_2011/vol1/Juska_Store_Supp.XLS

\section{References}

[1] R. Rizzuto, T. Pozzan, Microdomains of intracellular $\mathrm{Ca}^{2+}$ : molecular determinants and functional consequences, Physiol. Rev., 2006, 86, 369-408. http://dx.doi.org/10.1152/physrev.00004.2005

[2] M. Brini, E. Carafoli, Calcium pumps in health and disease, Physiol. Rev., 2009, 89, 1341-1378. http://dx.doi.org/10.1152/physrev.00032.2008

[3] J.A. Rosado, S.O. Sage, Farnesylcysteine analogues inhibit store-regulated $\mathrm{Ca}^{2+}$ entry in human platelets: evidence for involvement of small GTP-binding 
proteins and actin cytoskeleton, Biochem. J., 2000, 347, 183-192.

http://dx.doi.org/10.1042/0264-6021:3470183

[4] J.A. Rosado, S.O. Sage, Stewart O. Regulation of Plasma Membrane $\mathrm{Ca}^{2+}$-ATPase by Small GTPases and Phosphoinositides in Human Platelets, J. Biol. Chem., 2000, 275, 19529-19535. http://dx.doi.org/10.1074/jbc.M001319200

[5] S.L. Brownlow, A.G.S. Harper, M.T. Harper, S.O. Sage, A role for hTRPC1 and lipid raft domains in store-mediated calcium entry in human platelets, Cell Calcium, 2004, 35, 107-113. http://dx.doi.org/10.1016/j.ceca.2003.08.002

[6] A. Juška, I. Jardín, J.A. Rosado, Physical properties of two types of calcium stores and SERCAs in human platelets, Mol. Cell. Biochem., 2008, 311, 9-18. http://dx.doi.org/10.1007/s11010-007-9687-z

[7] P.C. Redondo, G.M. Salido, J.A. Pariente, S.O. Sage, J.A. Rosado, SERCA2b and 3 play a regulatory role in store-operated calcium entry in human platelets, Cell. Signal., 2008, 20, 337-346. http://dx.doi.org/10.1016/j.cellsig.2007.10.019

[8] B.V. Liengme (2003) A Guide to Microsoft Excel 2002 for Scientists and Engineers, Elsevier Butterworth-Heinemann, Amsterdam, Boston, Heidelberg, London, New York, Oxford, Paris, San Diego, San Francisco, Singapore, Sydney, Tokyo.

[9] A. Juška, Plasma membrane calcium pump and sodium-calcium exchanger in maintenance and control of calcium concentrations in platelets, Biochem. Biophys. Res. Commun., 2010, 392, 41-46. http://dx.doi.org/10.1016/j.bbrc.2009.12.153

[10] A. Juška, Calcium fluxes into and out of cytosol in human platelets: Analysis of experimental data, Biochem. Biophys. Res. Commun., 2011, 412, 537-542. http://dx.doi.org/10.1016/j.bbrc.2011.07.042

[11] E.C. Toescu, O.H. Petersen, Region-specific activity of the plasma membrane $\mathrm{Ca}^{2+}$ pump and delayed activation of $\mathrm{Ca}^{2+}$ entry characterize the polarized, agonist-evoked $\mathrm{Ca}^{2+}$ signals in exocrine cells, J. Biol. Chem., 1995, 270, 8528-8535. http://dx.doi.org/10.1074/jbc.270.15.8528

[12] A. Juška, Dynamics of calcium fluxes in nonexcitable cells: mathematical modeling, J. Membr. Biol., 2006, 211, 89-99. http://dx.doi.org/10.1007/s00232-005-7019-3

[13] D.E. Roberts, R. Bose, Molecular and Functional Characterization of the Human Platelet $\mathrm{Na}^{+} / \mathrm{Ca}^{2+}$ Exchangers, Br. J. Pharmacol., 2011. http://onlinelibrary.wiley.com/doi/10.1111/j.1476-53 81.2011.01600.x/abstract;jsessionid=2DAEA3A07B4 9F53A97845FEDADEFC599.d02t02.

[14] A. Juška, P.C. Redondo, J.A. Rosado, G.M. Salido, Dynamics of calcium fluxes in human platelets assessed in calcium-free medium, Biochem. Biophys. Res. Commun., 2005, 334, 779-786. http://dx.doi.org/10.1016/j.bbrc.2005.07.040 